\title{
Synthesis of New Anthihelmintic Analogs of Marine Natural Products*
}

\author{
Gloria Serra, Graciela Mahler, Sandra Gordon, Marcelo Incerti and Eduardo Manta \\ Cátedra de Química Farmacéutica. Facultad de Química, Universidad de la República.Av. General \\ Flores 2124, C.C. 1157, Montevideo, Uruguay
}

\begin{abstract}
The synthesis of new anthelmintic compounds derived from 2-amine-4-hydroxy$\delta$-valerolactams and 2,4-dialkylthiazoles is described. The synthetic procedures and biological activity data for these compounds will be presented.
\end{abstract}

Secondary metabolites of marine origin are a source of new molecular architectures with interesting and promising biological activities.

For some time our group has been carrying out a general program of discovery and development of new compounds with antihelmintic activity. For this purpose we have chosen a group of natural products isolated from sponges, which have displayed a very high antihelmintic activity. Such is the case of the Bengamides (1) and Micotiazol (2).

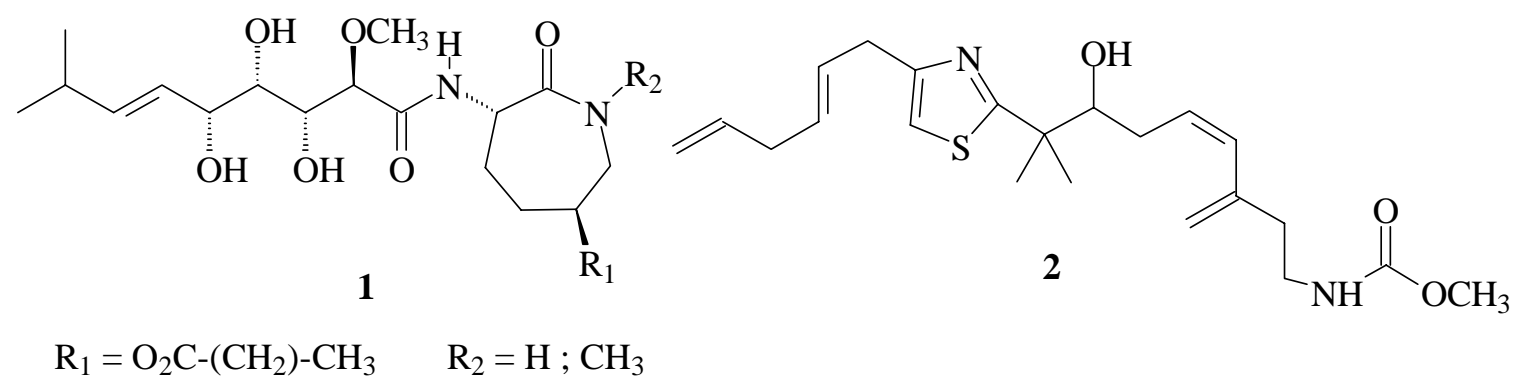

From a structural point of view these compounds share a common molecular pattern, in which a central heterocyclic ring, biogenetically derived from aninoacids, simultaneously bears sidechains with both lipo- and hydrophilic character. Molecular simplification of structures with proven biological activity is a classical tool used in Pharmaceutical Chemistry to obtain new lead compounds. This methodology was applied in our group starting from the basic structural patterns found in compounds of $\mathbf{1}$ and 2.

In this lecture we will first present our results when a group of derivatives 2-amino-4-hydroxy- $\delta$ valerolactam were prepared via a synthetic sequence involving lactonization followed by a lactonelactam exchange, as shown in Scheme 1. 
<smiles>[R1]N[C@H]1C[C@@H](O[R20])CN([R3])C1=O</smiles>

Scheme 1.

Second, we will describe the synthesis of 1,3-thiaza-2,4-disubstituted systems from acyclic precursors. We will discuss the results of different condensation and cyclodehydration methodologies, as well as the methods used to carry out controlled oxidations of the central heterocyclic system (Scheme 2).

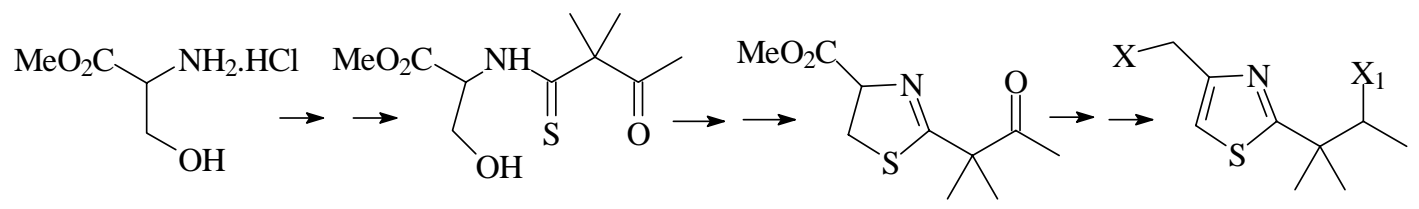

Scheme 2.

In all cases the antihelmintic activity of the synthesized compounds and the biological models used to evaluate these activities will be presented.

*Note: Translation by the Editorial Staff. 